\title{
A Heavenly Example of Scale Free Networks and Self-Organized Criticality
}

\author{
M. Paczuski* and D. Hughes \\ Mathematical Physics, Imperial College London, London, UK SW7 2BZ
}

\begin{abstract}
The sun provides an explosive, heavenly example of self-organized criticality. Sudden bursts of intense radiation emanate from rapid rearrangements of the magnetic field network in the corona. Avalanches are triggered by loops of flux that reconnect or snap into lower energy configurations when they are overly stressed. Our recent analysis of observational data reveals that the loops (links) and footpoints (nodes), where they attach on the photosphere, embody a scale free network. The statistics of the avalanches and of the network structure are unified through a simple dynamical model where the avalanches and network co-generate each other into a complex, critical state. This particular example points toward a general dynamical mechanism for self-generation of complex networks.
\end{abstract}

Key words: networks, self organized criticality, solar flares PACS: 96.60.Rd, 64.60.Lx

\section{Introduction}

Two generative themes to grasp complexity in its variety of manifestations confront us. One refers to a general dynamical principle, or how complexity is made. Another refers to ways of understanding inter-relations between parts of a complex system. We will leave aside the problem of defining complexity (1), which to date has been posed in terms of algorithms or information, rather than plausible dynamical laws.

The first theme we discuss is self-organized criticality (2; 3), which posits that the dynamics of slowly, driven dissipative systems impels them toward a

\footnotetext{
* Corresponding author

Email address: maya@ic.ac.uk (M. Paczuski).
} 
robust dynamical attractor that accommodates avalanches of all sizes. These avalanches, in turn, imprint long range correlations throughout the structure in which they occur. The physical system and the avalanches in it cogenerate each other through a long, historically contingent process into a complex entity. One cannot speak of one, the system, without the other, the avalanches.

Often, we are mislead by the intermittent occurrence of the avalanches into thinking of the two as being separate. Although most of the time, the system, such as the network of faults in the crust of the earth, appears static, one cannot forget that a dynamics, in this case seismic processes or earthquakes, must account for the current appearance of the network of faults as being a particular snapshot in geological time over which the crust of the earth, and network of faults has changed greatly. (Remembering this fact, one becomes skeptical of definitions of complexity which forget about the dynamics of the actual physical process that generates the ensemble of observed states.)

As the second theme, modern network theory has emerged as a powerful framework for describing many disparate systems ranging from the topology of the world wide web, to biological regulatory networks (4), or physical systems such as the coronal magnetic field (5). By using nodes as the basic objects, a great simplification in the description of the system can be obtained by only linking strongly interacting or correlated nodes. For example, since $N$ nodes can have at most $N(N-1) / 2$ links between them, pruning insignificant links can produce drastic simplifications, especially if the final number of links is of order $N$. Then, the network can be considered as the essential backbone of the inter-relationships between different parts of the system. In this way, networks may distill the main qualities, or most significant features, from the messy and hard-to-describe systems we generally refer to as complex.

Empirically, it has been observed that many networks are neither totally random nor completely ordered like a regular lattice. They lie in between, exhibiting strong heterogeneity from node to node. In fact, some networks are scale-free, in the sense that the number of links attached to any node is distributed as a power law, up to a cutoff determined by the total number of links in the network.

Various models of complex or scale-free networks have been proposed. In the preferential attachment model of Barabasi and Albert, a network grows by adding links to nodes with a probability proportional to the number of already existing links at that node $(\underline{6})$. A common feature of this and other models is that the process generating the network assures that a system wide network always exists. Furthermore, the number of nodes and links in the network are assumed to always grow.

However, one can imagine a different scenario where the number of nodes and 
links in the network are statistically stationary. Furthermore, and perhaps even more to the point, one can consider a situation where the system wide network only emerges after a transient self-organizing process. Before reaching the stationary state, no system wide network exists; it arises as a result of a long, historically contingent process. Such networks can be said to be selforganized networks, and in this case one can ask if self-organized criticality (SOC) provides a mechanism for the emergence of scale-free networks, out of a collection of uncorrelated random networks. This could happen just like a sandpile, with avalanches of all sizes, can be made out of a collection of uncorrelated sand grains by slowly adding them together without disturbing the system too much, for instance, by shaking the sandpile violently in a continuous fashion. We now give a specific example of a SOC mechanism for scale-free networks. We describe a naturally occuring physical system, namely the sun.

A complex interwoven network of magnetic fields threads the surface of the sun. Magnetic energy stored in the coronal network builds up due to turbulent plasma forces until stresses are suddenly released by a rapid reconfiguration of the magnetic field (7). This releases energy that is converted to radiation and observed as a solar flare. The probability distribution of flare energies is a featureless power law that spans more than eight orders of magnitude (8).

Lu and Hamilton first proposed that the corona is in a self-organized critical state (9), with avalanches of all sizes (2). However, SOC systems often show scale free behavior not only for their event statistics, but also for emergent spatial and temporal structures (2), as seen both in physical systems (e.g. (10)) and numerical models (e.g. (11)).

In fact, like flares, concentrations of magnetic flux on the photosphere (which is the visible surface of the sun) also exist on a wide variety of scales. The strongest concentrations are sunspots, which occur in active regions that may contain more than $10^{22} \mathrm{Mx}$. The smallest resolvable concentrations above the current resolution scale of $\approx 10^{16} \mathrm{Mx}$ are fragments. For many years, solar physicists have believed that at each scale a unique physical process is responsible for the dynamics and generation of magnetic concentrations, e.g. a "large scale dynamo" versus a "surface dynamo" etc.

Motivated by results from numerical simulations of a model, which is described later, we recently reanalyzed several previously published data sets reporting the distribution of concentration sizes (12). We just plotted the distribution using a double logarithmic scale instead of a log-linear one, as had been done previously. As shown in Fig. 1, we found that the distribution of magnetic flux concentration sizes is scale free over more than two decades, corresponding to the range of this particular measurement (5). 


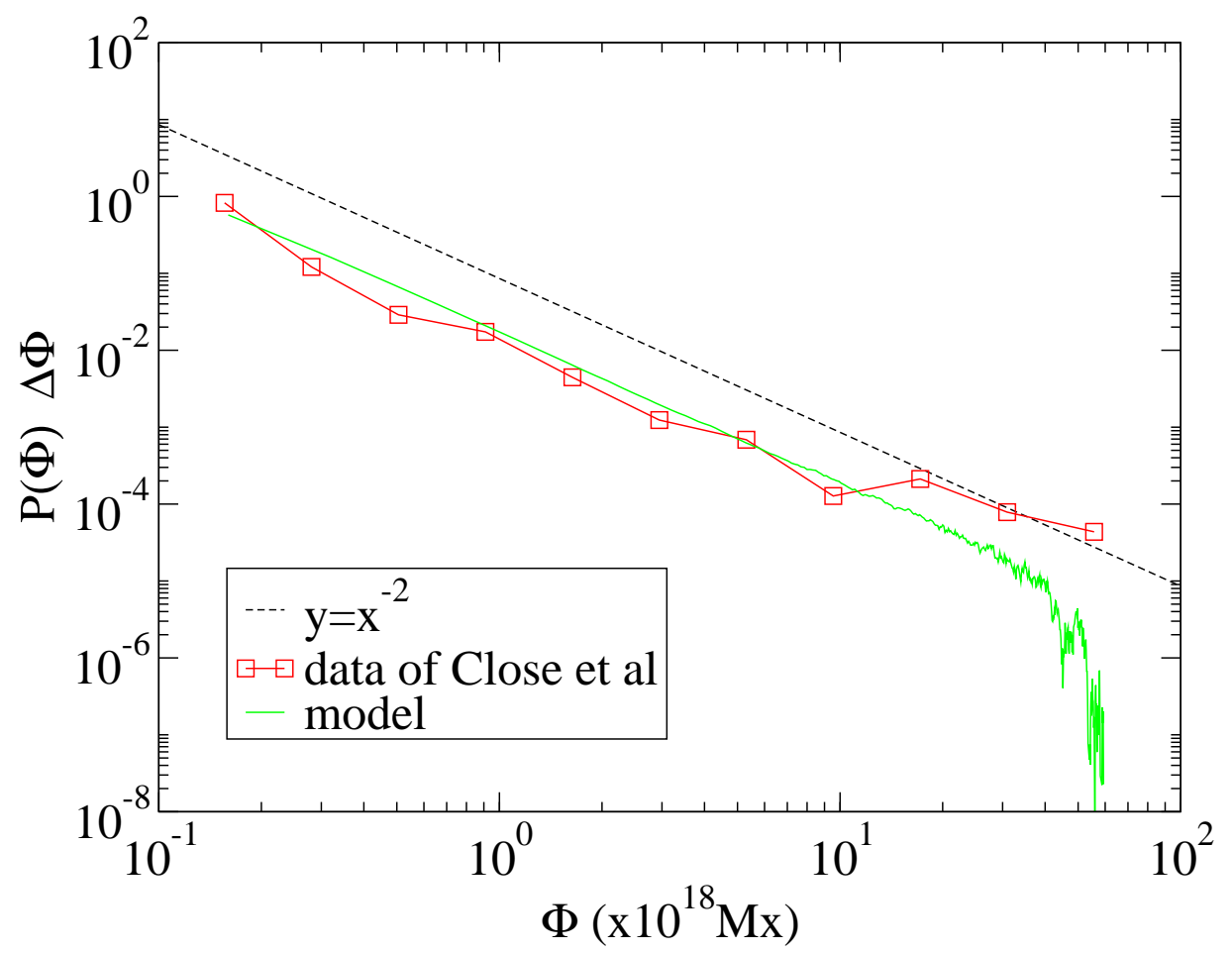

Fig. 1. The degree distribution of the magnetic network. The normalized number of magnetic concentrations in bins of size $\Delta \Phi=1.55 \times 10^{17} \mathrm{Mx}$ obtained by reanalyzing the measurement data originally shown in Figure 5 of Ref. (12). The model data shown represents the probability distribution, $P\left(k_{f o o t}\right)$, for number of loops, $k_{f o o t}$, connected to a footpoint. This has been rescaled so that one loop, $k_{\text {foot }}=1$, equals the minimum threshold of flux, $1.55 \times 10^{17} \mathrm{Mx}$

Of course the basic equations that describe the evolution of the magnetic field are continuous. However, the corona is a highly turbulent system and the dynamics of its magnetic field is intermittent. The field itself is concentrated into narrow regions and does not spread out uniformly. Observations of the solar corona reinforce this view, showing loops of hot plasma, emanating from magnetic concentrations, which are thought to trace out the paths of the magnetic flux tubes. So, although the fundamental equations of plasma physics are continuous and smooth, what we actually observe are well defined, discrete entities such as magnetic concentrations and flux tubes. We decided therefore to treat the coronal magnetic field as made up of discrete interacting loops (13).

The fundamental entity in the model is a directed loop that traces the midline of a flux tube, and is anchored to a flat surface at two opposite polarity footpoints. A footpoint locates the center of a magnetic concentration. A collection of these loops and their footpoints gives a distilled representation of the coronal magnetic field structure. Our network model is able to describe fields that are very complicated or interwoven. A snapshot of a configuration in the steady-state is shown in Fig. 2. The number of loops connecting any 


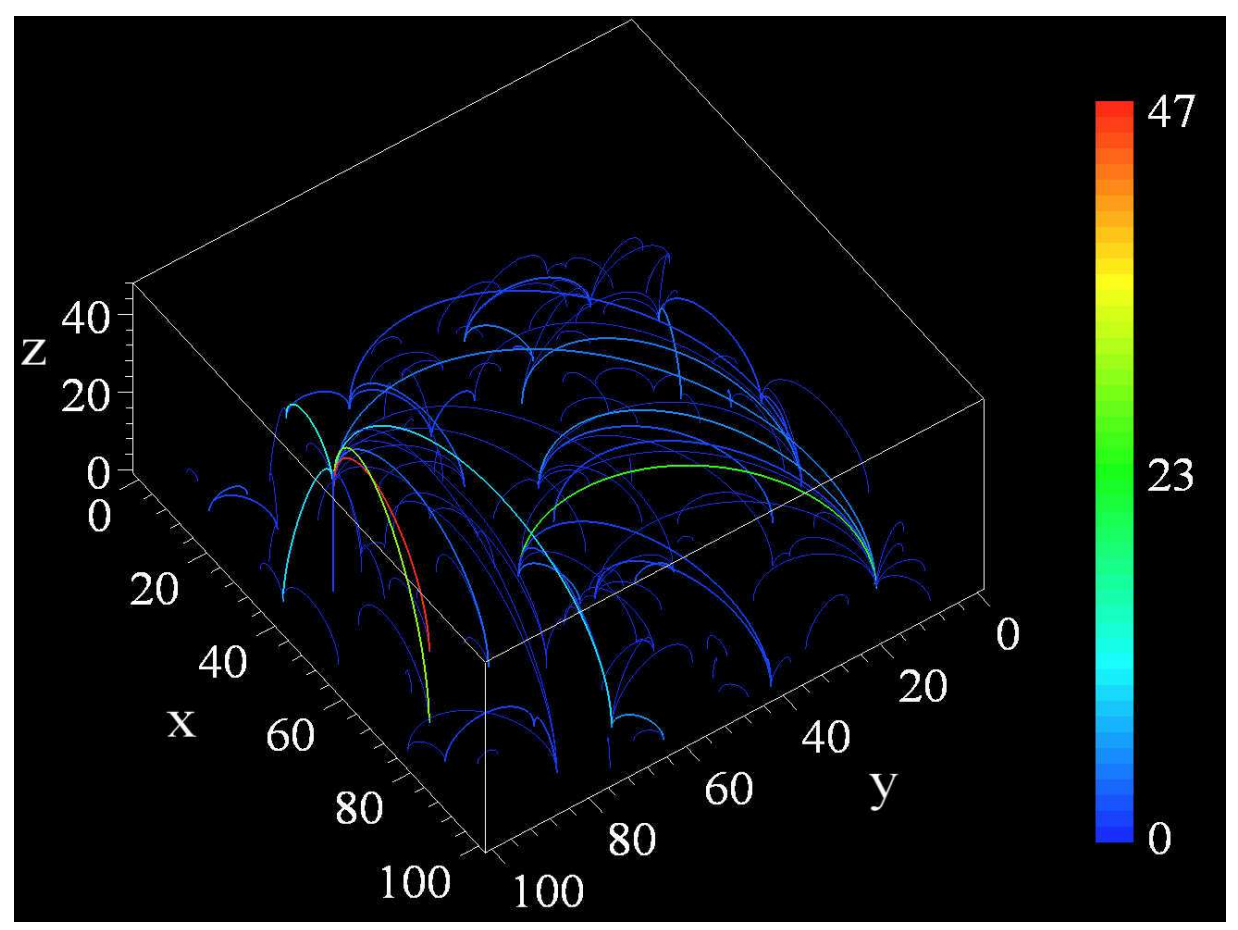

Fig. 2. Snapshot of loops in the steady state of the model. Footpoints lie in the (xy) plane and are linked by loops. The loops are colored to indicate the relative strength of the connection, as shown by the scale to the right. Note that there is a large range in the number of loops emanating from different footpoints, as well as a wide range of connection strengths.

pair of footpoints is indicated by a color-coding. It is evident that both the number of loops attached to a footpoint and the number of loops connecting any pair of footpoints vary over a broad range.

Loops injected at small length scales are stretched and shrunk as their footpoints diffuse over the surface. Nearby footpoints of the same polarity coalesce, to form magnetic fragments, which can themselves coalesce to form ever larger concentrations of flux, such as sunspots. Conversely, adjacent, opposite polarity footpoints cancel. Each loop carries a single unit of flux, and the magnetic field strength at a footpoint is given by the number of loops attached to it. The number of loops that share a given pair of footpoints measures the strength of the link.

Loops can reconnect when they collide in three dimensional space. The flux emerging from the positive footpoint of one of the reconnecting loops is then no longer constrained to end up at the other footpoint of the same loop, but may instead go to the negative footpoint of the other loop. This re-wiring process allows footpoints to exchange partners and reshapes the network, but it maintains the degree of each footpoint.

If rewiring occurs, it may happen that one or both loops need to cross some 
other loop. Thus a single reconnection between a pair of loops can trigger an avalanche of causally related reconnection events. Reconnections occur instantaneously compared to the diffusion of footpoints and injection of loops.

The system therefore evolves by a combination of slow diffusion of footpoints, occassional injection of new loops and rapid avalanches of reconnection. These avalanches punctuate quiet periods where the configuration of loops only changes gradually and slowly. Thus, a type of stick-slip dynamics is observed.

From arbitrary initial conditions, the system eventually reaches a steady state, where the cancellation processes on average balance the steady inflow of new loops. In the steady state we find that the system of loops and footpoints self organize into a scale free network (5). The number of loops, $k_{f o o t}$, connected to any footpoint is distributed as a power law

$$
P\left(k_{\text {foot }}\right) \sim k_{\text {foot }}^{-\gamma}, \quad \gamma \approx 2
$$

Clearly, each footpoint can be thought of as a node in the network and each loop as a link. Therefore, this distribution corresponds to the degree distribution of the network, and the exponent $\gamma$ is the exponent for the degree distribution.

There are a variety of ways to associate an energy with the loops, depending on their length, and how many loops connect a pair of footpoints. Reconnection will shorten loops and therefore liberate energy from the magnetic field. A cascade of reconnections represents a flare. Defining the energy to be only proportional to the total length of loops, the distribution of flare energies follows a power law (13). However, during the transient while the system organizes itself, the distribution of flare energies is not a power law. Thus, our model is a true SOC system.

We find two new quantities to characterize scale free networks. Numerical simulations of the model show that the strength of the link between two nodes, which is the number of loops connecting two nodes, is distributed as a power law, with an exponent $\alpha \geq \gamma$. In addition, the number of unique nodes linked to any specific node also exhibits scale free behaviour, with a different exponent, which is greater than $\alpha$.

To sum up, we have demonstrated that SOC is a mechanism for generating scale free networks. In our model the complex network which self-generates itself into a state with avalanches of all sizes is fully scale free. Not only is the degree distribution a power law, but so is the distribution of link strengths, the number of distinct nodes linked to any particular node, and the avalanches. The system wide network emerges only after a transient period where the avalanches and the network have cogenerated each other into a critical state. Before that happens, no system wide network exists and the avalanches are 
also limited by a scale smaller than the system size.

\section{References}

[1] P. Grassberger, Toward a quantitative theory of self-generated complexity, Int. J. Theor. Phys. 25 (1986) 907-938.

[2] P. Bak, C. Tang, K. Weisenfeld, Self-organized criticality: An explanation of 1/f noise, Phys. Rev. Lett. 59 (1987) 381-384.

[3] P. Bak, How Nature Works: The Science Of Self-Organized Criticality, Springer, New York, 1996.

[4] R. Albert, A. L. Barabási, Statistical mechanics of complex networks, Rev. Mod. Phys. 74 (2002) 47-97.

[5] D. Hughes, M. Paczuski, Scale-free magnetic networks: Comparing observational data with a self-organizing model of the coronal field, preprint astro-ph/0309230.

[6] A.-L. Barabasi, R. Albert, Emergence of scaling in random networks, Science 286 (1999) 509-512.

[7] E. Priest, T. Forbes, Magnetic Reconnection, Cambridge University Press, Cambridge, 2000.

[8] M. Aschwanden, T. D. Tarbell, R. W. Nightingale, C. J. Schrijver, A. Title, C. Kankelborg, P. Martens, H. Warren, Time variability of the "quiet" sun observed with TRACE. II. Physical parameters, temperature evolution, and energetics of extreme-ultraviolet nanoflares, Astrophys. J. 535 (2000) 1047-1065.

[9] E.T. Lu, R.J. Hamilton, Avalanches and the distribution of solar flares, Astrophys. J. Lett. 380 (1991) L89-L92.

[10] V. Frette, K. Christensen, A. Malthe-Sørenssen, J. Feder, T. Jøssang, P. Meakin, Avalanche dynamics in a pile of rice, Nature 379 (1996) 4952.

[11] D. Hughes, M. Paczuski, Large scale structures, symmetry, and universality in sandpiles, Phys. Rev. Lett. 88 (2002) 054302.

[12] R. Close, C. Parnell, D. MacKay, E. Priest, Statistical flux tube properties of 3d magnetic carpet fields, Sol. Phys. 212 (2003) 251-275.

[13] D. Hughes, M. Paczuski, R. O. Dendy, P. Helander, K. G. McClements, Solar flares as cascades of reconnecting magnetic loops, Phys. Rev. Lett. 90 (2003) 131101. 